\title{
Thyroglobulin immunoreactivity in lymph node histiocytes: a potential diagnostic pitfall
}

\author{
L Venkatraman, P Maxwell, W G McCluggage
}

\begin{abstract}
Aims-Strong thyroglobulin immunoreactivity within sinus histiocytes in a lymph node draining a papillary thyroid carcinoma was observed in a recent case. This prompted the investigation of whether thyroglobulin immunoreactivity is common in regional lymph nodes in cases of thyroid malignancy.

Methods-Eighty seven lymph nodes were studied from 21 cases of thyroid malignancy. These comprised papillary carcinoma $(n=12)$, follicular carcinoma $(\mathbf{n}=4)$, medullary carcinoma $(\mathbf{n}=3)$, and one case each of squamous and anaplastic carcinoma. Eleven cervical lymph nodes from patients with no evidence of thyroid disease were included as controls. Sections were stained with a monoclonal antibody against thyroglobulin.

Results-In the cases of thyroid malignancy, 32 of 87 lymph nodes showed positive staining for thyroglobulin of histiocytes within the subcapsular and medullary sinuses. In an additional four cases, there was positive staining of lymph within lymphatic channels. Positivity was present in at least one node in 15 of 21 cases. There was no positivity in the control cases. There was no correlation between the size of the primary tumour and the presence of thyroglobulin positivity.

Conclusions-Positive staining with antithyroglobulin occurs not uncommonly in sinus histiocytes in lymph nodes draining thyroid tumours. This positivity could be the result of the destruction of normal thyroid follicles, with the release of thyroglobulin, which is taken up by histiocytes, which subsequently drain to local lymph nodes. Pathologists should be aware of this phenomenon and should be careful not to interpret this as metastatic tumour.

(f Clin Pathol 2001;54:314-316)
\end{abstract}

Keywords: thyroid; thyroglobulin; immunohistochemistry

Immunocytochemistry and Molecular Pathology Laboratory,

Royal Group of Hospitals Trust, Belfast, Northern Ireland, UK

P Maxwell

Correspondence to: Dr Venkatraman glenn.mccluggage@ bll.n-i.nhs.uk

Accepted for publication 17 August 2000

Papillary carcinoma is the most common primary thyroid malignancy. Cervical lymph node metastasis may be present in up to $54 \%$ of cases at some stage in the evolution of the disease, ${ }^{1}$ and the presence or absence of local metastasis at the time of initial surgery is one of the most important prognostic parameters. In most cases, nodal metastasis is easily recognisable morphologically. However, in some cases there may be morphological doubt, for instance when nodal metastasis undergoes cystic degeneration to such an extent that all that remains is a cystic lesion lined by an attenuated layer of cells. In doubtful cases, thyroglobulin immunostaining can be used to confirm the presence of metastatic disease. Monoclonal antibodies against thyroglobulin have generally been considered to be specific for thyroid follicular epithelial cells.

Recently, we encountered a case of papillary thyroid carcinoma with cystic metastatic deposits within ipsilateral cervical lymph nodes and in which thyroglobulin staining was used to confirm the morphological suspicion of metastasis. In addition to positive staining within the tumour cells, sinusoidal histiocytes also showed immunoreactivity. We were unaware of reports of aberrant thyroglobulin reactivity in sinusoidal histiocytes in lymph nodes and this prompted us to investigate this further by carrying out thyroglobulin immunostaining on a series of draining lymph nodes in cases of thyroid malignancy.

\section{Materials and methods}

Eighty seven lymph nodes from 21 cases of thyroid malignancy were studied. The primary thyroid tumours comprised papillary carcinoma ( $n=12)$, follicular carcinoma $(n=4)$, medullary carcinoma $(n=3)$, and one case each of squamous and anaplastic carcinoma. Eleven cervical lymph nodes from patients without evidence of thyroid disease were also used as controls.

For immunohistochemistry, sections of $4 \mu \mathrm{m}$ thickness were cut from paraffin wax blocks and stained with antithyroglobulin monoclonal antibody (Dako, Glostrup, Denmark) at a mouse immunoglobulin concentration of $0.5 \mathrm{mg} / \mathrm{ml}$ (1/400). Negative controls were stained with IgG1 at an equivalent concentration. We used the Dako Envision detection system, which uses DAB as chromogen. All sections were counterstained with Harris's haematoxylin.

The biopsy reports were reviewed to ascertain whether the size of the thyroid tumours was correlated with the presence or absence of thyroglobulin immunoreactivity.

\section{Results} metastatic carcinoma. Thirty two of 87 nodes showed moderate to strong granular cytoplasmic staining for thyroglobulin of histiocytes within subcapsular and medullary sinuses (fig 1). This included 14 lymph nodes containing metastatic carcinoma. Positive staining was found in nodal histiocytes in 10 papillary, four
Of the 87 lymph nodes evaluated, 27 contained 


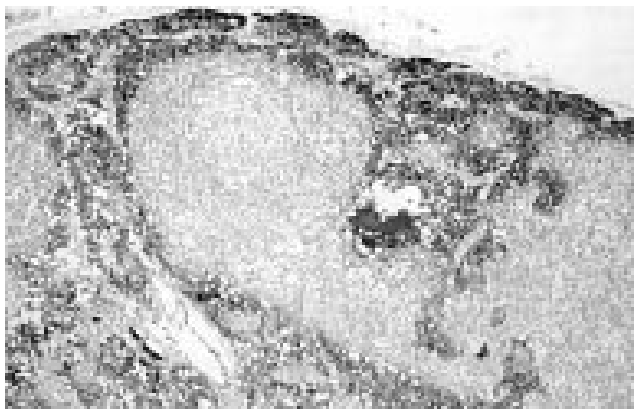

Figure 1 Strong positive staining for thyroglobulin of sinus histiocytes within the lymph node.

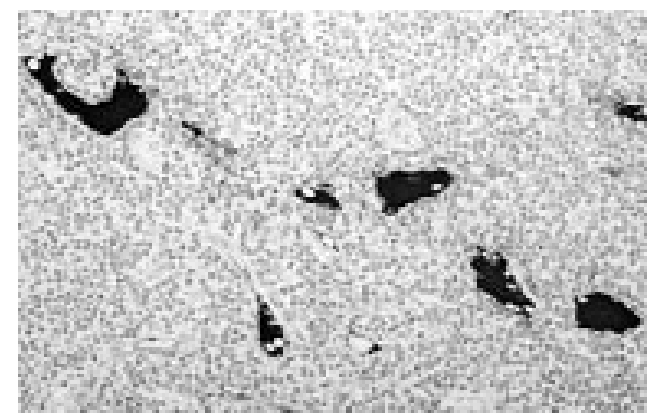

Figure 2 Strong positive staining for thyroglobulin of lymph within lymphatic channels.

follicular, and one anaplastic carcinoma. In four lymph nodes without demonstrable reactivity for thyroglobulin within histiocytes, lymph within lymphatic channels showed strong positivity (fig 2 ). This was also present in cases where there was thyroglobulin immunoreactivity within histiocytes. The 11 cervical lymph nodes from patients without evidence of thyroid disease were completely negative for thyroglobulin. There was no staining or, in a small number of cases, negligible background staining of collagen. There was no staining of negative controls.

The primary tumours in cases where lymph node positivity was present ranged from 0.3 to $7 \mathrm{~cm}$, with an average of $3.7 \mathrm{~cm}$. In cases where no nodal positivity was found, the tumours ranged from 0.2 to $13 \mathrm{~cm}$, with an average of $5.1 \mathrm{~cm}$.

\section{Discussion}

Our study demonstrates positive staining for thyroglobulin of histiocytes in a considerable proportion $(36.8 \%)$ of lymph nodes draining thyroid malignancies. At least one node was positive in 15 of 21 cases. This phenomenon, if erroneously interpreted, might result in an incorrect diagnosis of metastatic tumour. Local lymph node dissection is most commonly performed in suspected cases of papillary thyroid carcinoma and, if this diagnosis is known preoperatively (usually as a result of fine needle aspiration), the surgeon may search for enlarged lymph nodes in the region of the thyroid and, if found, they will be removed. In other fields of surgical pathology practice, there is an increasing focus on the detection of micrometastases within lymph nodes and, if thyroglobulin immunostaining is performed, and incorrectly interpreted, an erroneous diagnosis may be made. Nodal micrometastases most commonly involve the subcapsular sinus and, because thyroglobulin positive histiocytes are usually found here, this might cause further diagnostic confusion.

We are not aware of previous reports of thyroglobulin immunoreactivity of histiocytes in lymph nodes draining thyroid malignancies. However, it is recognised that tumours involving the thyroid, either primary or metastatic, which are not of follicular epithelial origin, may occasionally exhibit weak positivity. This is caused either by diffusion from normal thyroid follicular epithelium, with subsequent absorption by the tumour, or by destruction of normal thyroid with the release and uptake of thyroglobulin by the neoplasm. ${ }^{2-4}$ This might result in weak positive staining with antithyroglobulin antibodies and an inappropriate diagnosis of a tumour of thyroid follicular epithelium.

Positive staining for thyroglobulin was found mostly in lymph nodes of patients with papillary carcinoma but was also identified in cases of follicular and anaplastic carcinoma, suggesting that the phenomenon is not specific for any type of thyroid tumour. It is probable that the aberrant thyroglobulin positivity is the result of the destruction of normal thyroid by the tumour, with the release of thyroglobulin, which is taken up by histiocytes, which subsequently drain to local lymph nodes. This is supported by the location of the positive cells within subcapsular and medullary sinuses and by the presence of immunoreactivity in lymph within lymphatic channels. We have also seen thyroglobulin immunoreactivity within histiocytes in the thyroid gland in the vicinity of tumours, further supporting this hypothesis.

If aberrant thyroglobulin staining is caused by uptake by histiocytes secondary to tumour destruction of normal thyroid, it might be expected that positivity would be most common with large thyroid tumours, which result in considerable destruction. However, we could find no correlation between tumour size and the presence of thyroglobulin immunoreactivity within nodal histiocytes. This suggests that even with small tumours, local destruction might result in the uptake of thyroglobulin by histiocytes.

Our study did not investigate whether conditions other than tumours involving the thyroid could give rise to similar positivity of histiocytes for thyroglobulin within local lymph nodes. It would be interesting to investigate whether conditions such as lymphocytic thyroiditis can give rise to a similar phenomenon. In addition, with the increasing popularity of thyroid fine needle aspiration it could be speculated that this procedure might have similar effects.

In summary, we have demonstrated that thyroglobulin positivity is commonly found in histiocytes in lymph nodes draining thyroid tumours. Given the increasing propensity for 
the detection of nodal micrometastases, pathologists should be aware of this phenomenon if erroneous diagnoses are to be avoided.

1 Carcangiu ML, Zampi G, Pupi A, et al. Papillary carcinoma of the thyroid. A clinicopathologic study of 241 cases treated at the University of Florence, Italy. Cancer 1985;55:805-28
2 Wilson NW, Pambakian M, Richardson TC, et al. Epithelial markers in thyroid carcinoma: an immunoperoxidase study. Histopathology 1986;10:815-29.

3 Burt A, Goudie RB. Diagnosis of primary thyroid carcinoma by immunohistochemical demonstration of thyroglobulin. Histopathology 1979;3:279-86.

4 Carcangiu ML, Sibley RK, Rosai J. Clear cell change in primary thyroid tumors. A study of 38 cases. Am $\mathcal{F}$ Surg Pathol 1985;9:705-22.

\section{What's in the next issue}

\section{Future content}

See which articles have just been accepted for publication and preview the table of contents for the next issue a month before it is published

\section{wWw.jclinpath.com}

